\title{
Vehicle Relay Attack Avoidance Methods Using RF Signal Strength
}

\author{
Gyu-Ho Kim, Kwan-Hyung Lee, Shim-Soo Kim, Ju-Min Kim \\ Advanced Research Part, Daedong, Seoul, The Republic of Korea \\ Email: charran@dae-dong.biz, saicocoisa@hanyang.ac.kr, sskim@dae-dong.biz, jumin1985@dae-dong.biz
}

Received April 2013

\begin{abstract}
The number of passenger cars equipped with a smart key system continues to increase due to the convenience of the system. A smart key system allows the driver to enter and start a car without using a mechanical key through a wireless authentication process between the car and the key fob. Even though a smart key system has its own security scheme, it is vulnerable to the so-called relay attacks. In a relay attack, attackers with signal relaying devices enter and start a car by relaying signals from the car to the owner's fob. In this study, a method to detect a relay attack is proposed. The signal strength is used to determine whether the signal received is from the fob or the attacker's relaying devices. Our results show that relay attacks can be avoided by using the proposed method.
\end{abstract}

Keywords: Authentication; Relay Attack; Signal Strength; Smart Key System

\section{Introduction}

Since the introduction of the smart key at the present, the number of smart key installations has gradually increased due to the convenience of the device. A smart key system provides a driver with the ability to enter and start a car without using a mechanical key through a wireless authentication process. Even though a smart key system has its own security scheme, it is vulnerable to relay attacks. Tests on cars with smart key systems indicate that relay attacks can easily be performed by relaying a low frequency (LF) signal from the car through a relay attack device to the car's key fob. Most vehicles are susceptible to these relay attacks, regardless of the original equipment manufacturer (OEM). In this study, relay attack tests were performed on nine different cars from seven OEMs to confirm the potential for these attacks. From the data, an algorithm was developed to deter unauthorized entry using a threshold received signal strength indication (RSSI) value for the RF signal from the key fob.

\section{Overview of Smart Key Systems}

\subsection{Definition}

A smart key system is the equipment that allows a driver to enter and start a car through the process of authentication using fobs registered in the car. Using this system, the driver can control the door, trunk, and alarm by pressing the button on the fob from a distance. Smart key sys- tems have two main functions, listed below. Smart key system is the equipment that allows the driver to enter and start the car through the process of authentication with fobs registered in the car or allows the driver to control door, trunk, and alarm by pressing the button on the fob from a distance. Smart key system has the following two main functions.

\subsection{Functions}

The passive-entry passive-start (PEPS) feature is one of the functions of smart key systems that allows the driver to enter and start the car. If the driver triggers the door handle, then a LF signal (challenge signal) is transmitted from the car to the fob. The fob responds to the car (response signal) by sending a radio frequency (RF) signal. The car decodes the RF signal received from the fob and checks to see whether or not the fob is registered. If the driver triggers the door handle, then the door locks when the driver leaves the car. Relay attack problems commonly occur during the PEPS operation.

Remote keyless entry (RKE) is the second function of smart key systems that allows the driver to control the car from a distance. If the driver presses the button on the fob, then the car doors automatically lock or unlock.

\subsection{Types of Relay Attacks}

A relay attack is divided into LF and RF relay attacks, depending on which type $f$ signal is relayed through the 
relay attack devices. A LF relay attack sends a LF signal from the car to the fob. A RF relay attack relays both a LF signal from the car and a RF signal from the fob to the car. For a RF relay attack, the possible attack distance is longer (up to $1 \mathrm{~km}$ ) because a RF signal is also relayed. In this paper, we concentrated solely on a LF relay attack (Figures 1 and 2).

\subsection{Concept of LF Relay Attack Devices}

- LF relay attack devices located between the car and the fob amplify and transfer the LF signal that is sent when the driver triggers the door handle. Once the fob receives this LF signal, it verifies the information and responds to the car. The car receives this signal and confirms the information and then locks or unlocks the door.

- Relay attack devices are composed of two components: RA1 and RA2. RA1 is responsible for receiv- ing the LF signal from the car and transmitting it to RA2. RA2 is responsible for receiving the signal from RA1 and transmitting it to the fob.

- A $2.4 \mathrm{GHz}$ module is commonly used to transfer the signal between RA1 and RA2

\subsection{Performance of LF Relay Attack Devices}

Relay attack tests were performed on nine different cars from seven OEMs to confirm the potential for relay attacks in these vehicles. Our results showed that most cars with smart key systems were vulnerable to relay attacks (Table 1).

\subsection{Relay Attack Test Results}

- For the test, the LF frequency, supplier and relay attack vulnerability of each OEM were investigated

- Both passive entry and/or passive start were possible.

- OEM E's car $\eta$ had no passive entry option.

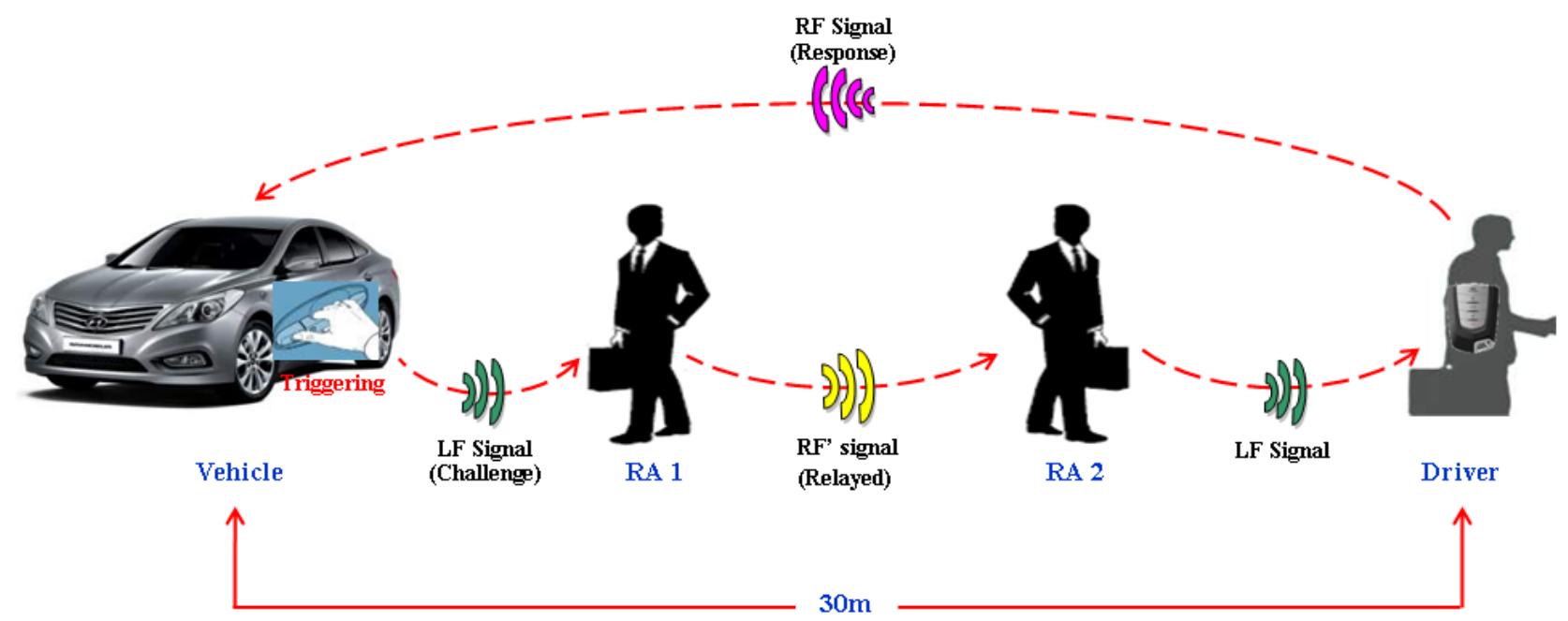

Figure 1. Concepts of a low frequency (LF) relay attack

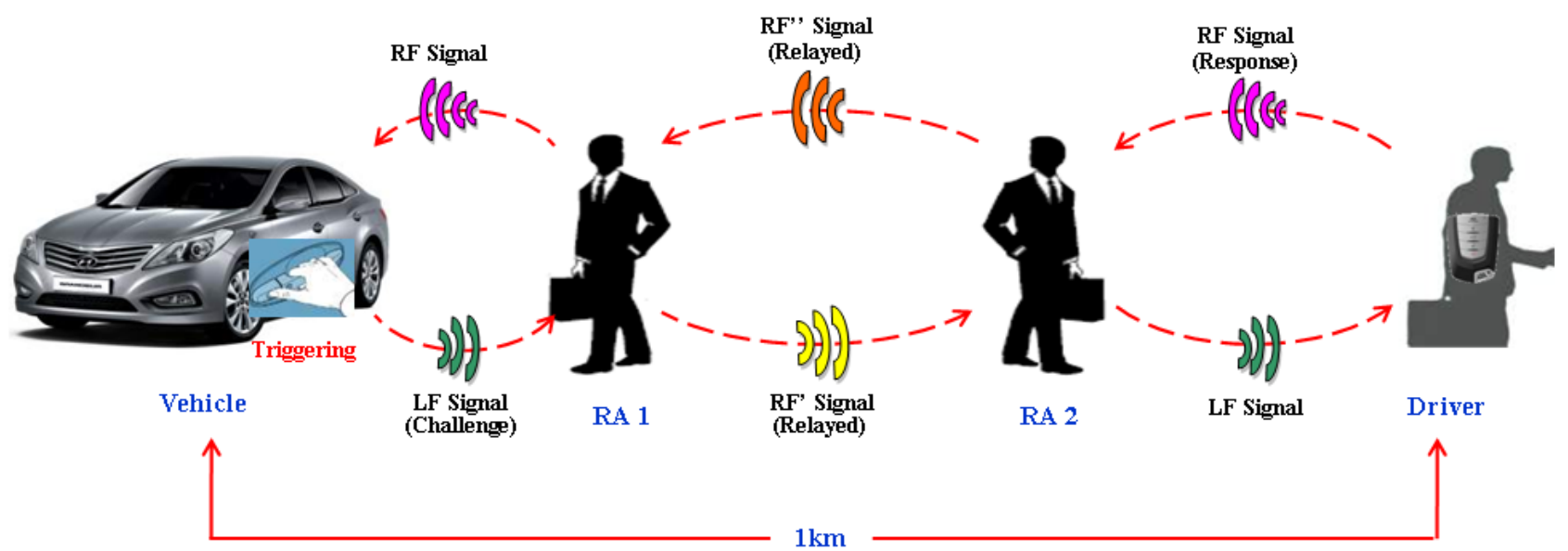

Figure 2. Concepts of a radio frequency (RF) relay attack. 
Table 1. Low frequency (LF) relay attack test results.

\begin{tabular}{|c|c|c|c|c|c|c|c|}
\hline \multirow{2}{*}{ No. } & \multirow{2}{*}{ OEM } & \multirow{2}{*}{ Vehicle } & \multirow{2}{*}{ LF Frequency (KHz) } & \multirow{2}{*}{ Supplier } & \multicolumn{2}{|c|}{ Test Results } & \multirow{2}{*}{ Comment } \\
\hline & & & & & $\mathrm{PE}$ & SP & \\
\hline \multirow{3}{*}{1} & & $\alpha$ & 134 & $\mathrm{~d}$ & $P$ & $\mathrm{P}$ & \\
\hline & A & $\alpha$ & 134 & d & $\mathrm{P}$ & $\mathrm{P}$ & \\
\hline & & $\gamma$ & 125 & c & $\mathrm{P}$ & $\mathrm{P}$ & \\
\hline 2 & B & $\delta$ & 125 & c & $\mathrm{P}$ & $\mathrm{P}$ & \\
\hline 3 & $\mathrm{C}$ & $\varepsilon$ & 125 & $\mathrm{w}$ & $\mathrm{P}$ & $\mathrm{P}$ & \\
\hline 4 & $\mathrm{D}$ & $\zeta$ & 134 & d & $\mathrm{P}$ & $P$ & \\
\hline 5 & $\mathrm{E}$ & $\eta$ & 125 & $\mathrm{~h}$ & - & $P$ & No PE Option \\
\hline 6 & $\mathrm{~F}$ & $\theta$ & 125 & g & $\mathrm{P}$ & $\mathrm{P}$ & \\
\hline 7 & G & $\alpha$ & 125 & c & $\mathrm{P}$ & $\mathrm{P}$ & \\
\hline
\end{tabular}

In the table1, P: Possible, I: Impossible, PE: Passive Entry, PS: Passive Start.

- Our test results showed that relay attacks were possible within a distance determined by the output power of the fob (100 m maximum).

- Because the attackers could move out of the driver's view, it was difficult for the driver to recognize that the car's security had been compromised, if someone implemented a relay attack

- Solutions for LF relay attack problems are proposed based on the test results.

- An algorithm is proposed based on the RSSI value of the fob's RF signal to prevent car thefts based on fatal relay attacks.

\subsection{Algorithm Based on the Fob'S RF Signal RSSI Value}

To implement an algorithm using the fob's RF signal RSSI value, the point at which a fob's output power is fixed at $0 \mathrm{dBm}$ for both PEPS and RKE operations is considered. Usually, the PEPS feature is actuated as the driver approaches the car. The signal from a relay attack device travels from some distance. Thus, if the RSSI value of the RF signal is lower than a given threshold value, then it can be regarded as a relay attack. This feature of the algorithm is summarized below.

1) Algorithm using fob's RF signal RSSI value

a) This algorithm based on fob's RF signal RSSI value can determine the proximity of the fob using the RSSI value of the RF signal.

b) If a car receives an RF signal from a relayed LF signal from a distance, then the RSSI value is lower due to signal attenuation.

c) Therefore, if the RSSI value is lower than a predetermined threshold value, the vehicle determines that the signal corresponds to a relay attack and ignores it, even though it has been received.

2) Data acquisition process for each test scenario

a) To determine the threshold value to identify a relay attack, the RSSI value of the RF signal was measured in accordance with the environment of the fob.

b) Two methods were used to measure the RSSI values. First, the RSSI value was determined with respect to the distance and direction of the key fob from the car. Second, the RSSI value was determined with respect to the direction of the fob in relation to the car as well as the voltage state for a fixed distance of $2 \mathrm{~m}$.

c) To consider the attenuation of the RF signal of the fob as the battery discharges, the RSSI value of the RF signal was measured in response to the state of the fob battery for each direction. The distance at which the RSSI value was measured was initially $2 \mathrm{~m}$, and the gradually increased to $50 \mathrm{~m}$ in $5-\mathrm{m}$ increments, starting from $5 \mathrm{~m}$. The direction between the fob antenna and the car was varied between to $0^{\circ}, 90^{\circ}$ and $180^{\circ}$. We did not measure the RSSI value at $270^{\circ}$ because when viewed from the position of the car, the value was equal to $90^{\circ}$ value.

d) For a fixed distance of $2 \mathrm{~m}$, the RSSI was measured while the fob's battery voltage was reduced from $3.0 \mathrm{~V}$ to $2.0 \mathrm{~V}$ in $0.2-\mathrm{V}$ increments, for various directions between the fob antenna and the car of $0^{\circ}, 90^{\circ}$ and $180^{\circ}$. These tests were used to confirm the minimum RSSI value within the distance required for normal Passive Entry.

e) Testing was performed at these test sites: and open site, an indoor parking lot, and an outdoor parking lot. The test results represent an average of the results obtained from the three test sites (Figures 3 and $\mathbf{4}$ ).

\subsection{Basis for Threshold Value Determination}

The threshold value used to identify a relay attack must be properly selected. If the threshold is too high, then it could impede normal PEPS operation. The results indicated that the RSSI value gradually decreases as the distance increases. Assuming that the distance over which a driver can recognize a relay attack is $2 \mathrm{~m}$, the minimum RSSI value was acquired at a fob voltage of $2 \mathrm{~V}$ for $180^{\circ}$ between the fob antenna and the car (Figures $\mathbf{5}$ and $\mathbf{6}$ ). 

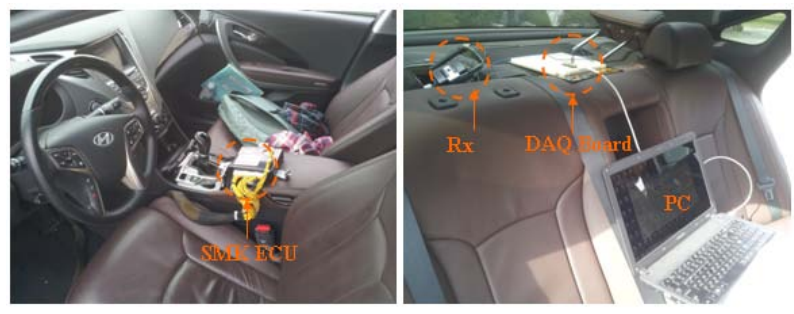

Figure 3. Data acquisition equipment.
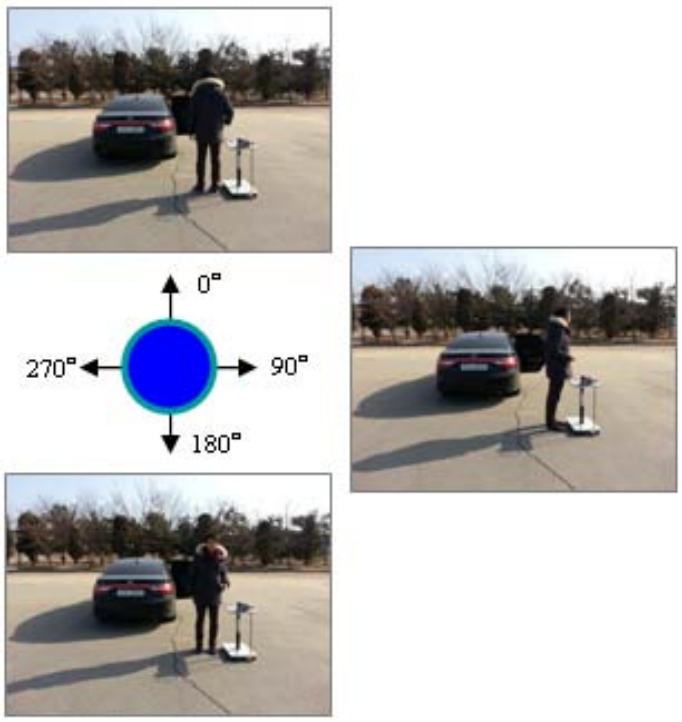

Figure 4. Data acquisition method.

\subsection{Algorithm flow Chart and Verification}

The proposed algorithm was successfully applied to a smart key system. When the vehicle received the RF signal, if the RSSI value was higher than the threshold value, then the door was unlocked; otherwise, the door remained locked (Figure 7).

\section{Conclusions}

To avoid LF relay attacks, it is necessary to determine whether the key is close to or far from the car based on the received RF signal strength. We developed an algorithm that measured the RSSI value and compared this with a threshold value. If the RSSI value was lower than the determined threshold value, then a relay attack was perceived by the system, and the RF signal was ignored. Thus, locked car doors remained locked, preventing entry by the attacker.

The results of our tests on nine vehicles from seven OEMs indicated that normal PEPS operation would be possible for distances of $<2 \mathrm{~m}$ (PEPS operation was not observed for distances exceeding $2 \mathrm{~m}$ ). However, if a relay attack device RA1 is located near the car, and an RF signal is transmitted from the fob, then a relay attack could occur. In this scenario, an attack is possible be-

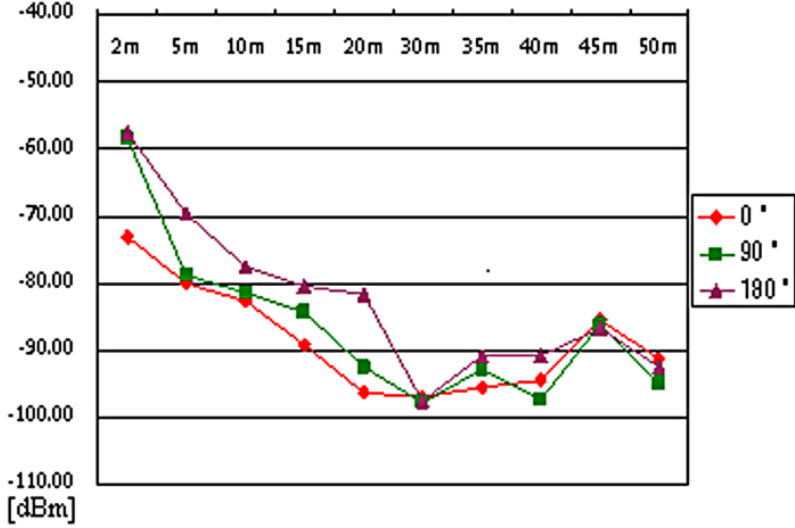

Figure 5. RSSI value as a function of distance and direction.

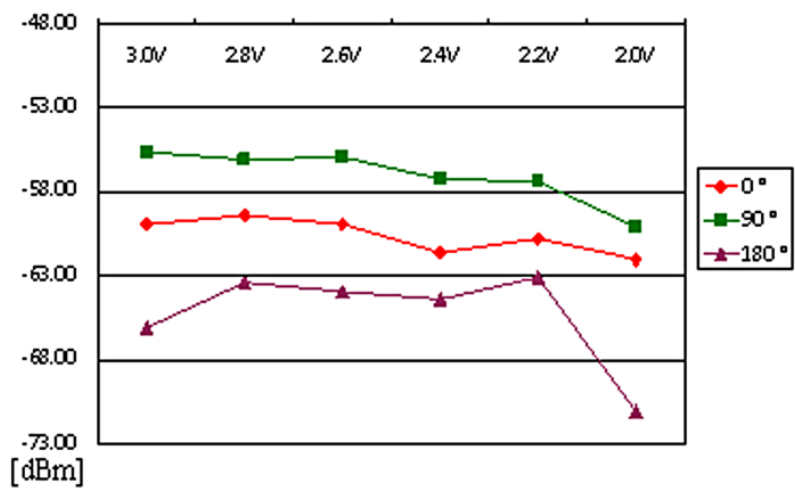

Figure 6. RSSI value as a function of direction and voltage.

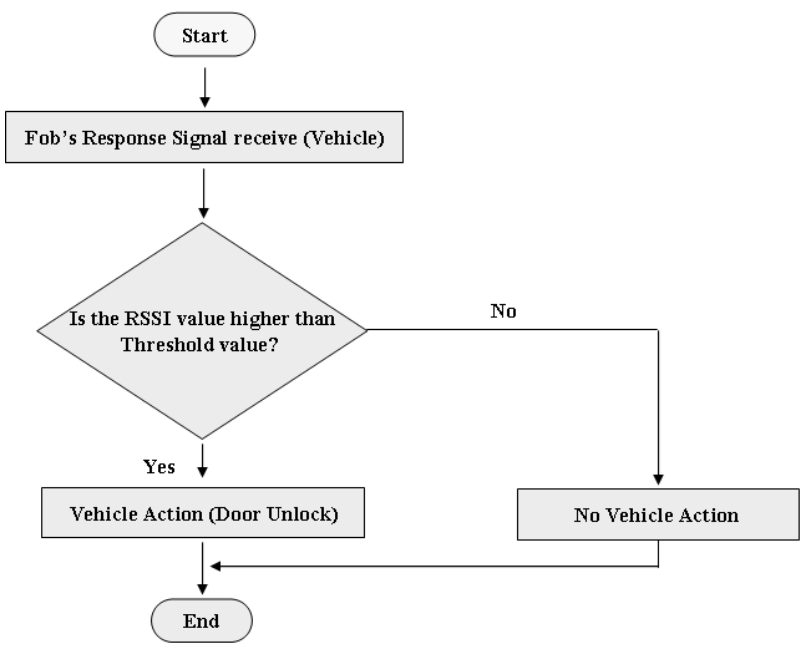

Figure 7. Flow chart of relay attack avoidance algorithm.

cause the relay attack device can possibly transfer an RF signal that was greater than the threshold value. The current authentication method uses LF and RF one-way communication. Two-way authentication uses LF one-way and RF two-way communication. In future studies, multi-channel, divided packet transfer may also be considered with RF two-way authentication. 


\section{REFERENCES}

[1] A. I. Alrabady, "Security of Passive Access Vehicle," Submitted to the Graduate School of Wayne State University, Detroit, Michan.

[2] A. I. Alrabady and S. M. Mahmud, "Some Attacks against Vehicle's Passive Entry Security Systems and Their Solutions," IEEE Transactions on Vehicular Technology, Vol. 52, No. 2, March 2003. http://dx.doi.org/10.1109/TVT.2003.808759

[3] A. I. Alrabady and S. M. Mahmud, "Analysis of Attacks against the Security of Keyless-Entry Systems for Vehicles and Suggestions for Improved Designs," IEEE Transactions on Vehicular Technology, Vol. 54, No. 1, January 2005.

http://dx.doi.org/10.1109/TVT.2004.838829

[4] A. Francillon, B. Danev, S. Capkun, "Relay Attacks on Passive Keyless Entry and Start Systems in Modern Cars," Department of Computer Science ETH Zurich 8092 Zurich, Switzerland.

[5] F. Stajano, F.-L. Wong and B. Christianson, "Multichannel Protocols to Prevent Relay Attack," University of Cambridge Computer Laboratory, Cambridge, United King- dom, DSO National Laboratories, Singapore, University of Hertfordshire, School of Computer Science, Hatfield, United Kingdom.

[6] A. Leitch, "Electronic Communication System, in Particular Access Control System for Passive Keyless Entry, as well as Method for Detecting a Relay Attack Thereon,” US Patent No. 0206989A1, 2009

[7] R. Ghabra, Y. Luo, J. Nantz and R. O. King, "Method for Apparatus for an Anti-theft System against Radio Relay Attack in Passive Keyless Entry/Start Systems," US Patent No. 0143500A1, 2008

[8] R. Ghabra, N. Yakovenko and H. W. Girard, "Method and System of determining and Preventing Relay Attack for Passive Entry/Start System,” US Patent No. 0321154 A1, 2008

[9] H. Masudaya, "Passive Entry with Anti-theft Function," US Patent No. 0168997A1, 2005

[10] E. Perraud and M. Burri, "Passive Response Communication System,” US Patent No. 6992568B2, 2006

[11] C. Tieman, J. Coudre and T. P. Oman "Vehicle Security System and Method of Operation Based on a Nomadic device Location,” US Patent No. 201202. 